\title{
Interferon Induced Transmembrane Protein-1 Gene Expression is a Biomarker for Early Detection of Invasive Potential of Oral Squamous Cell Carcinomas
}

\author{
Arvind Ramanathan
}

\begin{abstract}
Background: Early detection of malignant transformation with expression biomarkers has significant potential to improve the survival rate of patients as such biomarkers enable prediction of progression and assess sensitivity to chemotherapy. The expression of interferon inducible transmembrane protein 1 (IFITM1) has been associated with early invasion events in several carcinomas, including head and neck cancers, and hence has been proposed as a novel candidate biomarker. As the incidence of oral squamous cell carcinoma (OSCC) is highest in the Indian population, we sought to investigate: 1) the expression pattern of IFITM1 in OSCC tissue samples obtained from Indian patients of Dravidian origin; and 2) the possibility of using IFITM1 expression as a potential biomarker. Materials and Methods: Total RNA extracted from thirty eight OSCC biopsy samples was subjected to semi-quantitative RT-PCR with IFITM1 and GAPDH specific primers. Results: Of the thirty eight OSCC samples that were analyzed, IFITM1 overexpression was identified in fifteen (39\%). Seven expressed a low level, while the remainder expressed high level of IFITM1. Conclusions: The overexpression of IFITM1 in OSCC samples indicates that IFITM1 may be explored for the possibility of use as a high confidence diagnostic biomarker in oral cancers. To the best of our knowledge, this is the first time that IFITM1 overexpression is being reported in Indian OSCC samples.
\end{abstract}

Keywords: IFITM1 expression - oral carcinoma - over expression - biomarker - Indian cancers

Asian Pac J Cancer Prev, 17 (4), 2297-2299

\section{Introduction}

Interferons (IFN) are multifunctional cytokines that are produced by host cells to contain microbial infections. Upon secretion of IFN, a host of interferon-stimulated genes (ISGs) are upregulated via JAK / STAT (Laljee et al., 2013). More than 400 ISGs have been reported so far (Andersen et al., 2006a), of which some of them like ISG15 for example, have been shown to be expressed in carcinomas. Although the exact mechanism that triggers the expression of ISGs in tumor cells is not clear, several studies continue to identify candidate ISGs in diverse tumors (Andersen et al., 2006b; Bektas et al., 2008; Rajkumar et al., 2011) including oral squamous cell carcinoma (OSCC) tissues (Lang-Ming et al., 2009; Laljee et al., 2013).

The IFN- inducible transmembrane protein 1 (IFITM1) is one such candidate gene that belongs to a family of IFN-inducible transmembrane protein family that consists of IFITM2, IFITM3 and IFITM5 (Hatano $\mathrm{H}$ et al., 2008). IFITM1 gene is located on chromosome 11 at $11 \mathrm{p} 15.5$ region and produces a $17 \mathrm{kDa}$ transmembrane protein. The first identified function of the protein product was that it acts as a transducer of antiproliferative and homotypic adhesion signals in lymphocytes and inhibits early stages of viral replication (Lee et al., 2012; Cheon et al., 2014). However, following its identification in whole genome expression analysis of tumor cells, the role of IFITM1 began to be extensively studied that consequently led to the identification of its role in the promotion of cell invasion (Hatano et al., 2008; Lee et al., 2012) and drug response (Moy et al., 2013; Choi et al., 2015). More recently, several studies have shown association between higher expression of IFITM1 and progression of cancers of diverse tissues including head and neck cancer, serous ovarian cancer, gastric cancer, colorectal cancer, leukemia, lymphoma and cervical squamous cell carcinoma (Hatano et al., 2008; Cheon et al., 2014).

Despite therapeutic advances, the 5-year survival rate of patients with oral squamous cell carcinoma (OSCC) remains at $\sim 50 \%$, and hence the incidence of OSCC continues to rise every year especially in India (Mishra et al., 2014). One fundamental factor that contributes to such a high incidence is due to OSCCs being diagnosed at advanced stages. Early detection of OSCCs by screening of precancerous lesions and conditions for expression biomarkers has potential to greatly improvise the survival rate. However, this requires a reliable biomarker that

${ }^{1}$ Human Genetics Laboratory, Sree Balaji Medical and Dental College and Hospital, Bharath University, Narayanapuram, Pallikaranai, Chennai, Tamil Nadu, India*For correspondence: drarvindram@yahoo.co.in 
may be confidently used in a particular population. IFITM1 appears to be a high confident biomarker as its expression has been positively associated with invasion and progression in several tumors including head and neck squamous cell carcinomas of Japanese patients (Hatano et al., 2008). Although the expression of IFITM1 has been confirmed in head and neck carcinomas, the Indian Genome Variation Consortium has identified the genetic background of the Indian subpopulation to be distinct from HapMap populations (IJVC, 2008; Crena et al., 2015). As a result, both the mutation profile and expression pattern of disease causing genes is likely to distinct as well. This prompted us to design the present study to investigate the expression pattern of IFITM1 in OSCCs of Indian patients by semi-quantitative RT-PCR.

\section{Materials and Methods}

\section{Clinical samples}

In order to reduce confounding effects that may arise due to variation in genetic background, patients from a Tamil Nadu of Dravidian race alone were included in the present study. Tumor biopsies from thirty eight OSCC patients with well differentiated oral squamous cell carcinoma were surgically excised. A part of sample was stored in RNA Save for expression analysis, while the other part was used for histopathological examination. Informed consent from all the patients, and the study was approved by institutional ethics committee.

\section{Tissue storage, RNA extraction and RT-PCR}

At the time of RNA extraction, tumor biopsy samples stored RNA Save reagent (cat \# 01-891, Biological Industries) were washed twice with sterile 1X PBS (Phosphate Buffered Saline) and processed for RNA extraction by trizol protocol (TRIzol ${ }^{\circledR}$ Plus RNA Purification System, cat \# 12183555, Ambion) as recommended by the manufacturer. Following quantification, 500ng of total RNA from each of the sample was reverse transcribed with random primers (High capacity RNA to cDNA kit, cat \# 4387406, Life Technologies). Polymerase chain reaction was performed on all samples with IFITM1F/R and GAPDH-F/R primers (primer sequences are available upon request). The samples were amplified under the following conditions: initial denaturation at $94^{\circ} \mathrm{C}$ for $2 \mathrm{~min}$, followed by a 35 cycles of $94^{\circ} \mathrm{C}$ for $45 \mathrm{sec}, 55^{\circ} \mathrm{C}$ for $45 \mathrm{sec}$, and $72^{\circ} \mathrm{C}$ for $45 \mathrm{sec}$, with a final extension at $72^{\circ} \mathrm{C}$ for $5 \mathrm{~min}$.

\section{Quantification of IFITM1 expression}

$15 \mu 1$ of PCR amplified samples were subjected to electrophoresis in a $1.2 \%$ agarose gel, and images were captured in a gel documentation unit (Gelstan, MEDICCARE scientific supplies, Chennai, India). The images were then analyzed with NIH Image J software under default settings to quantify the intensity of IFITM1 and GAPDH amplicons. The scanned values of IFITM1 were normalized against GAPDH and plotted with excel software.

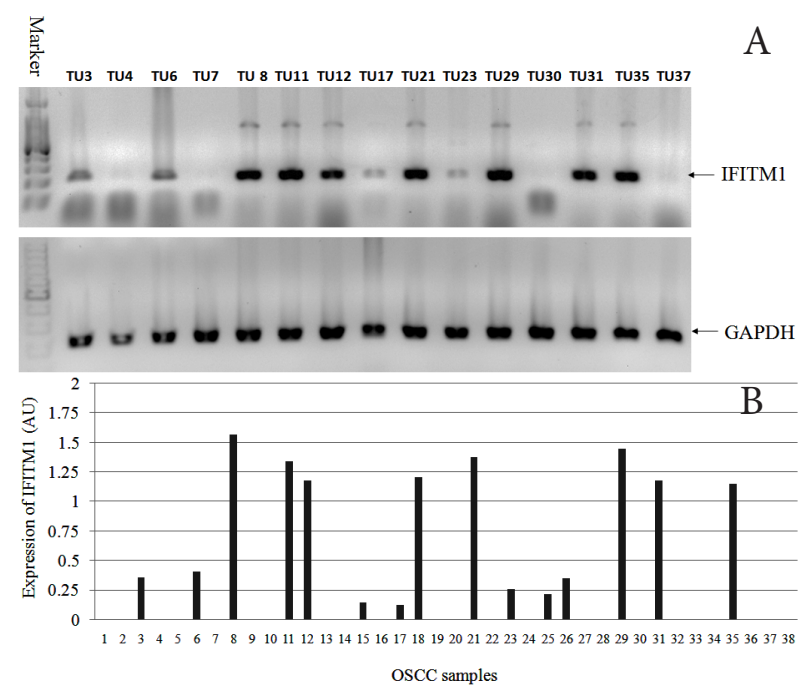

Figure 1. Overexpression of IFITM1 in OSCC

Samples. A. Representative image of semi-quantitative RTPCR of OSCC samples. Note that the expression level of IFITM1 varies among samples. B. Graphical representation of varied or lack of expression of IFITM1 among thirty eight OSCC samples. Seven samples showed low IFITM1 expression (up to 0.5AU), while eight showed high expression (greater than 1AU). AU Arbitrary Units

\section{Results}

In order to understand whether IFITM1 was overexpressed in OSCCs of Indian patients of Dravidian origin, we analyzed total RNA isolated from thirty eight histologically confirmed tumor biopsies by semiquantitative RT-PCR. Agarose gel analysis of the RT-PCR amplicons showed overexpression of IFITM1 in fifteen of thirty eight OSCC samples (39\%) (Figure 1A). The gel images of both IFITM1 and the house keeping gene - GAPDH, which served as control for amplification in each sample, were quantified with NIH Image J software. The values of IFITM1 were normalized against GAPDH values to obtain actual expression of IFITM1 in each sample and were plotted (figure 1B). Analysis of the data showed stratification of the tumor samples into two groups: low expressers - samples that expressed IFITM1 up to $0.5 \mathrm{AU}$ and high expressers - those with above $1 \mathrm{AU}$. In accordance to the above stratification, seven OSCC samples were identified as low expressers, while the rest of eight samples were identified as high expressers.

\section{Discussion}

IFITM1 is a novel candidate gene that was recently found to promote invasion and migration of HNSCC cells by immunohistochemical analysis of invasive tumor samples (Hatano et al., 2008). Interestingly, the overexpression of IFITM1 was observed focally in invasive front of early invasive lesions. Further in vitro analysis confirmed the role of IFITM1 in tumor invasion, and identified the gene as the second most overexpressed genes in highly invasive clones (Hatano et al., 2008). Based on these findings, we investigated the expression of IFITM1 in thirty eight OSCC tissue biopsy samples by semi-quantitative RT-PCR analysis, as OSCCs is highly 
prevalent in the Indian population. Overexpression of IFITM1 indeed was observed in 39\% (15/38) of OSCC tumor biopsy samples, of which seven samples expressed low levels, while eight of them expressed high levels. The IFITM1 specific band was confirmed by direct sequencing of the RT-PCR amplicons (data not shown). The reason for variation in the level of expression of IFITM1 observed the tumor samples is not clear. However such variation in the level of expression has been observed in other IFN induced genes such as ISG15 for example (Laljee RP et al, 2013) in OSCCs, carcinoma of breast $(66 \%-76 \%)$ (Betkas N et al., 2008) and bladder (93\% - 98\%) (Andersen JB et al. 2006b). Interestingly IFITM1 expression level was observed to be markedly different in the intestinal and diffuse types of gastric cancer, with intestinal type exhibiting higher expression than the diffuse type (Lee et al., 2012). Based on the expression pattern and immunohistochemical association, it was suggested that level of expression of IFITM1 influences the development and progression of intestinal-type gastric cancer. Investigating the reason for differential expression pattern resulted in the identification of CpG hypomethylation at -200 to 400 (exon1 - intron 1) region of IFITM1 gene in high expressers (Lee et al., 2012). We believe that such a mechanism may have existed in OSCC samples analyzed in the present study causing differential expression of IFITM1. However, whether the differential expression in OSCC was associated with its invasion potential remains to be investigated.

Studies have also shown the presence of IFITM1 in inflammatory cells adjacent to tumor regions, postulating the possibility of inflammatory interaction between the tumor and its microenvironment induced by the cytokines. IFITM1 has also been suggested to be a pro-inflammatory mediator as it is induced in response to signal from CD147 in macrophages (Kim et al., 2010). Inflammatory areas are frequently observed in oral precancerous lesions such as erythroplakia for example (Das et al., 2015). As OSCCs also arise as a result of malignant transformation of oral precancerous lesions with an estimated range from $1.4 \%$ to $36 \%$ at an interval of 1 to 30 years (Yardimci et al., 2014), expression analysis of IFITM1 combined with hypermethylation analysis in these lesions will provide in-depth understanding of its early role in invasion and transformation. Nevertheless, the finding of IFITM1 overexpression in 39\% of OSCC samples, strongly indicates it as a potential biomarker to understand the invasion and progression potential of OSCC tumors. The findings gains further clinical significance, as targeting IFN induced genes has been shown to sensitize tumors to chemotherapy (Moy et al., 2013; Choi et al., 2015).

\section{References}

Andersen JB, Hassel BA (2006a). The interferon regulated ubiquitin-like protein, ISG15, in tumorigenesis: Friend or foe. Cytokine Growth Factor Reviews, 17, 411-421.

Andersen JB, Aaboe M, Borden EC, et al (2006b). Stageassociated overexpression of the ubiquitin-like protein, ISG15, in bladder cancer. Br J Cancer, 94, 1465-71.

Bektas N, Noetzel E, Veeck J et al (2008). The ubiquitinlike molecule interferon-stimulated gene 15 (ISG15) is a potential prognostic marker in human breast cancer. Breast Cancer Res, 10, 58.

Cheon H, Borden EC, Stark GR (2014). Interferons and their stimulated genes in the tumor microenvironment. Semin Oncol, 41, 156-73.

Choi HJ, Lui A, Ogony J, Jan R, Sims PJ, Lewis-Wambi J (2015). Targeting interferon response genes sensitizes aromatase inhibitor resistant breast cancer cells to estrogen-induced cell death. Breast Cancer Res, 17, 6 .

Crena J, Subramanian S, Victor DJ, Gnana PP, Ramanathan A (2015). Single nucleotide polymorphism at -1087 locus of interleukin-10 gene promoter is associated with severe chronic periodontitis in nonsmoking patients. Eur J Dent, 9, 387-93.

Das S, Shenoy S (2015). Sneak peek into tobacco habits and associated insidious oral lesions in an odisha sample population. Asian Pac J Cancer Prev, 16, 7007-9.

Hatano H, Kudo Y, Ogawa I, Tsunematsu T, et al (2008). IFNinduced transmembrane protein 1 promotes invasion at early stage of head and neck cancer progression. Clin Cancer Res, 14, 6097-105.

Indian Genome Variation Consortium (2008). Genetic landscape of the people of India: a canvas for disease gene exploration. J Genet, 87, 3-20.

Kim JY, Kim H, Suk K, Lee WH (2010). Activation of CD147 with cyclophilin a induces the expression of IFITM1 through ERK and PI3K in THP-1 cells. Mediators Inflamm, 2010, 821940 .

Laljee RP, Muddaiah S, Salagundi B, et al (2013). Interferon stimulated gene-ISG15 is a potential diagnostic biomarker in oral squamous cell carcinomas. Asian Pac J Cancer Prev, 14, 1147-50.

Lang-Ming C, Chien-Wei L, Kai-Ping C et al (2009). Enhanced interferon signaling pathway in oral cancer revealed by quantitative proteome analysis of microdissected specimens using 16o/18o labeling and integrated two-dimensional LCESI-MALDI Tandem MS. Mol Cell Proteomics, 8, 1453-74.

Lee J, Goh SH, Song N, et al (2012). Overexpression of IFITM1 has clinicopathologic effects on gastric cancer and is regulated by an epigenetic mechanism. Am J Pathol, 181, 43-52.

Mishra A, Meherotra R (2014). Head and neck cancer: global burden and regional trends in India. Asian Pac J Cancer Prev, 15, 537-50.

Moy I, Lin Z, Rademaker AW, et al (2013). Expression of estrogen-related gene markers in breast cancer tissue predicts aromatase inhibitor responsiveness. PLoS One, 8, 77543.

Rajkumar T, Sabitha K, Vijayalakshmi N, et al (2011). Identification and validation of genes involved in cercival tumorigenesis. BMC Cancer, 11, 80.

Yardimci G., Kutlubay Z, Engin B, Tuzun Y (2014). Precancerous lesions of oral mucosa. World J Clin Cases, 2, 866-872. 